\title{
Critical Evaluation of Binary Rare-Earth Phase Diagrams
}

\author{
By K. A. Gschneidner, Jr. and F.W. Calderwood \\ lowa State University
}

\section{Introduction}

The purpose of this project is to present critical evaluations of the known rare-earth binary phase diagrams. In addition to reviewing the known phase relationships, crystallographic and thermodynamic data where available will be used in the evaluations. Other physical property data that may be appropriate also will be used. These would include: hardness, electrical resistivity, superconductivity, magnetic susceptibility, NMR, Mössbauer, etc. measurements.

The term "rare earths" is taken to include the elements scandium, yttrium, and the "lanthanides" (lanthanum, cerium, praseodymium, neodymium, promethium, samarium, europium, gadolinium, terbium, dysprosium, holmium, erbium, thulium, ytterbium, and lutetium). This definition of the term"rare earths" is the same as used by the International Union of Pure and Applied Chemistry (IUPAC).

\section{Ideal Liquid and Solid Solution Behavior of the Intra-Rare-Earth Alloys}

As is well known, the neighboring lanthanide elements are quite similar in their physical and chemical properties (except for those which depend directly on the $4 f$ electrons) because of the filling of the inner $4 f$ level as the nuclear charge increases when going from one element to the next in increasing atomic number. This similarity has an important effect on the intra-rare-earth binary alloys, i.e., if neighboring or near-neighboring elements are alloyed, they behave as ideal alloys at high temperatures. Thus, there is no measurable difference in the liquidus and solidus temperature, i.e., the two-phase liquid plus solid region is quite narrow (a few degrees or less). Furthermore, the liquidus/solidus line is a straight line (within experimental error) connecting the respective melting points of the pure metals. The same is true for the double closepacked hexagonal (dcph), face-centered cubic (fcc), or close-packed hexagonal (cph) to body-centered cubic (bcc) transformation that occurs near the melting point. This behavior only holds if the two components are trivalent and have atomic numbers within $\sim \pm 4$ of each other. This has been observed experimentally for the $\mathrm{Tb}-\mathrm{Ho}, \mathrm{Tb}-\mathrm{Er}$, Dy-Ho, Dy-Er, and Ho-Er systems [73Spe]. The other important factor is that this behavior is only observed if high purity metals (>99.9 at.\% pure) are used. Another verification of this is found in the study of the physical proper- ties of pure mischmetal (approximate composition is $50 \% \mathrm{Ce}, 25 \% \mathrm{La}, 15 \% \mathrm{Nd}, 5 \% \mathrm{Pr}$, and $5 \%$ other REM) by Palmer et al. [82Pal], who found that if the mischmetal did not contain any heavy lanthanides and the cerium contents were less than $40 \%$, then the observed melting and transition temperatures agreed with those calculated from the sum of the products of the transition and melting points of the pure metals times their respective atomic fractions.

Because many of the reported phase diagrams were based on studies using impure metals, the phase diagrams reported here have been modified to show this ideal behavior. If scientists use commercially available metals, the experimental alloying behavior they experience may not be exactly the same as expected from the phase diagrams given here.

\section{Non-Ideal Behavior}

For binary alloys involving yttrium or scandium with one of the other rare-earth metals, or if the atomic numbers of the two lanthanide metals differ by more than \pm 4 , then departures from ideal behavior, as discussed above, are to be expected.The departures are expected to vary depending upon the alloying partners - the further apart the two metals are in the periodic table, the greater the difference. An example is the Er-Y system, which was also studied by Spedding et al. [73Spe]. They found that both the transition and melting point variations with alloying exhibited a minimum departure from ideal behavior, but no separation of the liquidus/solidus and the two solvus lines was observed.

Non-ideal behaviors also are expected for rare-earth metals that are alloyed with the two divalent lanthanide metals - europium and ytterbium. Cerium and samarium also may be anomalous because these two metals have a tendency to exhibit valence states other than the normal $3^{+}$state $\left(2^{+}\right.$for $\mathrm{Sm}$ and $4^{+}$for $\left.\mathrm{Ce}\right)$. However, the mischmetal study reported by Palmer et al. [82Pal] did not indicate any anomalous behavior for cerium in this polycomponent system.

\section{Cited References}

73Spe: F.H. Spedding, B. Sandeen and B.J. Beaudry, J. LessCommon Met., 31, p 1 (1973).

82Pal: P.E. Palmer, H. R. Burkholder, B.J. Beaudry and K. A. Gschneidner, Jr., J. Less-Common Met, , to be published in 1982. 